\title{
DERECHOS HUMANOS Y LAS ENTIDADES NO HUMANAS: ALGUNAS REFLEXIONES SOBRE LAS CORPORACIONES
}

\author{
HUMAN RIGHTS AND NON HUMAN ENTITIES: A FEW THOUGHTS ON \\ CORPORATIONS
}

\author{
Migle Laukyte \\ Universitat Pompeu Fabra
}

Fecha de recepción: 18-10-18

Fecha de aceptación: 22-4-20

Resumen: El trabajo se ocupa de la cuestión de si los derechos humanos pueden atribuirse a las personas no humanas, y en especifico, a las corporaciones. Se analiza tal posibilidad (a) mirando a la relación entre los derechos humanos y las corporaciones a través de la libertad de empresa como derecho fundamental, (b) observando el proceso de "humanización" de la corporación en los tribunales, y (c) explicando que reconocer los derechos humanos a no humanos no siempre es prejudicial para las personas humanas.

Abstract: The paper focuses on the question whether the human rights could be ascribed to non human persons, and in particular, to corporations. This question is analyzed by (a) exploring the link between human rights and corporations through the freedom to conduct a business as a fundamental right, by (b) looking into the process of corporate "humanization" carried out in the courts, and by (c) explaining how ascribing human rights to non humans does not necessarily mean lessen the position of human beings.

Palabras clave: corporación, derechos humanos, persona humana, personalidad, humanización

Keywords: corporation, human rights, human being, personhood, humanization 


\section{INTRODUCCIÓN}

Los tratados y acuerdos internacionales sobre los derechos humanos generalmente no se refieren a las entidades no humanas como titulares de dichos derechos. ¿Y por qué tendrían? No humanos, por definición, no tienen por qué tener derechos humanos. Una rara excepción a este razonamiento es la Convención Europea de Derechos Humanos de 1953: con otros pocos tratados más, dicha Convención reconoce que las organizaciones no gubernamentales (ONGs) pueden demandar por ser víctimas de las violaciones de los derechos humanos ${ }^{1}$.

El presente trabajo no se centra en las ONGs en sí mismas, sino en las corporaciones, es decir, en las grandes empresas multinacionales, que por su poder decisorio, económico y financiero se han convertido en actores internacionales en el verdadero sentido del término ${ }^{2}$. Aun más, en su papel de actores internacionales, muchas veces son la fuente de las amenazas de los derechos humanos y muy raramente las víctimas de la violación de los mismos. Pero entre esos dos papeles -el de violador siempre y nunca la víctima- hay espacio para otros escenarios, a los que está dedicado este trabajo.

El interés de esta relación entre las corporaciones y los derechos humanos viene reforzado por lo que está sucediendo en los tribunales de todo el mundo: las corporaciones obtienen más derechos que antes fueron solo y exclusivamente de los humanos. Cómo explicar esta tendencia es uno de los motivos que ha animado esta investigación.

El presente trabajo está organizado en tres partes. Una primera parte, donde se enfoca la libertad de empresa como el puente entre los derechos humanos y la corporación moderna. Además, se observa la particularidad del derecho de la libertad de empresa: es uno de los pocos derechos que los seres humanos y entidades no humanas (las corporaciones) comparten sin limitaciones.

A continuación, se analiza la tendencia -observable en distintos contextos no solo de filosofía del derecho sino también y sobre todo en los contex-

1 Carta de los Derechos Fundamentales de la Unión Europea (2012/C326/02) también garantiza los derechos de las personas jurídicas, como, por ejemplo, derecho a acceder a los documentos (art. 42), derecho a someter al Defensor del pueblo Europeo los casos de mala administración (art. 43), y el derecho de petición antes del Parlamento Europeo (art. 44).

2 Mikler por ejemplo, explica el fenómeno corporativo como nacional en su identidad, regional en sus operaciones y global en sus intereses, pero el enfoque más importante sigue el de su impacto político: J. MIKLER, The Political Power of Global Corporations, Polity Press, Cambridge, 2018. 
tos jurisprudenciales- de humanizar las corporaciones, conscientemente o no, asignándoles cada vez más atributos humanos, y, en consecuencia, cada vez más derechos. Parece que el derecho humano de la libertad de empresa no marca los límites de los derechos humanos que podrían ser extendidos más allá de los seres humanos.

En una última parte final, se defenderá la tesis de que aunque esta tendencia ha sido - iy continua siendo!- disruptiva, hay algo positivo en esta tendencia: reconocer los derechos humanos a los no humanos puede ser una medida necesaria para garantizar los mismos a los humanos. Por consiguiente, extender los derechos humanos no necesariamente pone en peligro a las personas, y el presente trabajo se dedica a explorar esta posibilidad.

\section{TRES PREMISAS}

Para empezar, son tres las premisas para que el razonamiento que se expone seguidamente pueda desarrollarse sin malentendidos.

La primera premisa es que mientras la discusión se enfoque en las corporaciones como un ejemplo de las entidades o personas no humanas, esto no quiere decir que las corporaciones son las únicas a las que el ordenamiento jurídico reconoce derechos y deberes. Es decir, que no son únicos ejemplos de entidades con personalidad jurídica. Al contrario, hay muchísimos otros ejemplos, como por ejemplo las fundaciones, cooperativas, partidos políticos, las ya mencionadas ONGs, los sindicatos, las organizaciones internacionales y muchos otros supuestos. Por lo tanto, la corporación en este trabajo tiene el papel de suplente más representativo de todas otras entidades no humanas, pero, además, a diferencia de las arriba mencionadas, sucede muchas veces que tienen recursos económicos muy elevados y superiores a los de muchos países ${ }^{3}$.

La segunda premisa se refiere a lo que es una corporación. Desde el punto de vista histórico, cuando la idea de la personalidad corporativa moderna se desarrolló a finales del siglo XIX y a principios del siglo XX, no había un acuerdo -y sigue sin haberlo- sobre qué son las corporaciones: creaciones de

$3 \quad$ Ejemplar en este sentido es la investigación de J. Johnson: entre las 200 entidades más ricas en el mundo 157 son corporaciones y solo 43 son gobiernos: corporaciones como Apple, Walmart y Shell tienen más recursos que Suecia o el Bélgica. Por más detalles, véase J. JOHNSON, "157 of World's 200 Richest Entities are Corporations, Not Governments", Inequality.org, Oct. 19, 2018, disponible a https://inequality.org/research/richest-entities-corporations-governments/ 
ficción, personas particulares con derechos, colectivos de personas físicas, destinadas a proteger los derechos de sus accionistas y miembros individuales, o cualquier combinación de estas ideas ${ }^{4}$. Sin embargo, ahora mismo parece que se está concretando la idea de que no es tan importante lo que es una corporación, sino lo que la misma hace. Se acoge esta idea no solo porque, realmente, no hay una definición de corporación establecida y reconocida por la doctrina jurídica, sino porque el enfoque de esta discusión se pone en las consecuencias de sus actividades en y para la sociedad. Por la misma razón, a pesar de que la personalidad jurídica se reconoce a las corporaciones solo en el Derecho nacional, y por lo tanto no en derecho internacional ${ }^{5}$, también hay que tener en cuenta las corporaciones internacionales. Por lo tanto, en lugar de definir la corporación con una definición doctrinal, en este trabajo la corporación como concepto representa una idea nocional que se refiere a cualquier entidad comercial con personalidad jurídica, es decir, que existe y responde separadamente de sus accionistas, directivos, empleados o cualquier otra persona humana involucrada en su continuo funcionamiento y que además tiene un poder económico y social relevante ${ }^{6}$.

Esta descripción es suficientemente específica para identificar la corporación como una entidad no humana provista de una voluntad propia, que le permite actuar en el mundo social, político, económico y jurídico, pero, al mismo tiempo, es una noción suficientemente abierta para incluir una vasta gama de entidades que pueden ser locales, nacionales, internacionales, pero sobre todo, para los objetivos de este trabajo, de gran tamaño.

La tercera premisa es sobre el Derecho de referencia al plantear el discurso: el enfoque se centra en Europa y la jurisprudencia del Tribunal Europeo de Derechos Humanos (TEDH) como uno de los potentes instrumentos para

4 B. L. GARRETT, “The Constitutional Standing of Corporations", University of Pennsylvania Law Review, núm 63, 2014, pp. 95-164. Más sobre el tema de distintas teorías y funciones de las corporaciones, entre muchos otros, M. M. BLAIR, "Corporate Personhood and the Corporate Persona", University of Illinois Law Review, núm 3, 2013, pp. 785-820.

$5 \quad$ N J̈̈GERS, "The Legal Status of the Multinational Corporation Under International Law", en VV.AA., Human Rights Standards and the Responsibility of Transnational Corporations, edición de M. K. ADDO, Kluwer Law International, La Haya, 1999, pp. 260-270.

6 Más sobre el concepto de la corporación y la conexión entre la misma y la personalidad jurídica, entre otros, los clásicos de H. KELSEN, Teoria general del Derecho y del estado, Dykinson, $3^{\mathrm{a}}$ ed., Madrid, 2010, y F. W. MAITLAND, The Collected Papers of Frederic William Maitland, edición de H. A. L. FISHER, Cambridge UNiversity Press, Cambridge, 1911. 
garantizar los derechos humanos en la Unión Europea (UE) ${ }^{7}$. Ello no obsta a la existencia de algunas referencias a la jurisprudencia de EEUU y de otros países, así como a las decisiones de otros tribunales internacionales.

\section{LA LIBERTAD DE EMPRESA: MÁS ALLÁ DEL ENLACE ENTRE LOS DERECHOS HUMANOS Y LA CORPORACIÓN}

A pesar de que los conceptos de derechos humanos y de corporación pueden parecer muy distantes, hay un vínculo común subyacente entre los dos que testimonia una relación mucho más compleja y profunda entre esos conceptos, una relación que va más allá del enfrentamiento y la incompatibilidad.

En particular, la corporación moderna puede ser vista como el resultado de la realización de la libertad de empresa, la libertad que la Carta de los Derechos Fundamentales de la Unión Europea (La Carta) reconoce como derecho fundamental. La libertad de empresa es un derecho muy poco reconocido en los tratados de derechos humanos internacionales, o por lo menos reconocido no directamente. Por ejemplo, el Pacto Internacional de Derechos Económicos, Sociales y Culturales (adoptado en el 1966 y en vigor desde el 1976) se aproxima reconociendo el derecho a "la oportunidad de todos para ganar su vida mediante un trabajo libremente escogido o aceptado" (art. 6). Normalmente, la libertad de empresa es parte de las constituciones nacionales, como la de España cuyo art. 38 reconoce "la libertad de empresa en el marco de la economía de mercado. Los poderes públicos garantizan y protegen su ejercicio y la defensa de la productividad, de acuerdo con las exigencias de la economía general y, en su caso, de la planificación". En este sentido, es importante subrayar la posición de Tribunal Europeo de Justicia que ha establecido, ya desde hace muchos años en el caso Stauder v Ulm (29/69 [1969] ECR 419), que los derechos humanos que derivan de las tradiciones constitucionales de los países miembros, son parte del orden jurídico euro-

7 Véase N.-L. AROLD, The Legal Coulture of the European Court of Human Rights, Martinus Nijhoff Publishers, Leiden-Boston, 2007. Además, hay que aclarar también que $h u-$ manos en la expresión derechos humanos no lleva connotaciones del alto principio que normalmente esta expresión ha tenido: utilizo este termino sencillamente como un indicador de los derechos de los seres humanos porque en esta manera la expresión derechos humanos puede referirse a cualquier derecho reconocido a los humanos por cualquier gobierno de los Estados de Derecho. Se usa esta definición tan amplia intencionadamente para poder incluir sea los derechos humanos reconocidos a nivel internacional, sea los derechos civiles a nivel nacional. 
peo $^{8}$. Por lo tanto, la libertad de empresa, reconocida como derecho constitucional en la Constitución Española, es parte integrante del Derecho Europeo. Sin ninguna duda, hay otros derechos y libertades humanos que han influido directamente sobre el desarrollo de la corporación y han reforzado la libertad de empresa, como, por ejemplo, la libertad profesional y el derecho a trabajar (art. 15 de La Carta), el derecho a la propiedad (art. 17), y el derecho de establecimiento (art. 49 del Tratado de Funcionamiento de la Unión Europea) ${ }^{9}$. La conexión con los otros derechos humanos se entiende mejor si se tiene en cuenta que la libertad de empresa ha sido considerada como un derecho deseable, ya que su esencia es permitir, promover y favorecer el espíritu emprendedor y de la innovación, sea tanto a nivel individual como a nivel de la comunidad ${ }^{10}$. Ahora bien, si es el mismo derecho fundamental (libertad de empresa) sobre el cual se construye la base de la prosperidad individual y el crecimiento empresarial, cabe entender por qué la corporación puede ser considerada en el origen de libre desarrollo de la personalidad humana y por lo tanto es inseparable del concepto de la dignidad humana ${ }^{11}$.

Desde esta perspectiva, y teniendo en cuenta que la corporación forma parte de un proceso global de la prosperidad individual ${ }^{12}$, se puede observar la conexión entre los derechos humanos, la libertad de empresa y la corpora-

8 M. EVERSON y R. CORREIA GONÇALVES, "Article 16 - Freedom to Conduct a Business" en VV.AA., The EU Charter of Fundamental Rights: A Commentary, edición de S. PEERS, T. HERVEY, J. KENNER, A. WARD, Hart, Oxford-Portland, 2014, pp. 437-464.

9 Esta conexión entre la libertad de empresa y los derechos económicos y sociales la observa también G. CHANDLER, "Crafting a Human Rights Agenda for Business", en VV.AA., Human Rights Standards and the Responsibility of Transnational Corporations, edición de M. K. ADDO, Kluwer Law International, La Haya, 1999, pp. 39-45. Además hay que precisar que el derecho de establecimiento no es limitado solo para crear una corporación, sino que también permite de entrar en los negocios como autónomo o crear cualquier tipo de entidad comercial (art. 49 2nda parte). De hecho, la forma corporativa es solo una de muchas formas de hacer negocios, M. DAN-COHEN, Rights, Persons, and Organizations: A Legal Theory for Bureaucratic Society, Quid Pro Books, $2^{\mathrm{a}}$ ed., New Orleans, 2016.

10 AGENCIA EUROPEA DE LOS DERECHOS FUNDAMENTALES, Freedom to Conduct a Business: Exploring the Dimensions of a Fundamental Right, Oficina de publicaciones de la Union Europea, 2015.

11 Según M. EVERSON y R. CORREIA GONÇALVES, "Article 16 - Freedom to Conduct a Business" cit., la libertad de empresa también representa una parte integrante de los esfuerzos por parte de UE de crear una economía política que vaya más allá de los nacionalismos.

12 I. GARCÍA VICTORIA, La Libertad de Empresa: Un Terrible Derecho?, Centro de Estudios Politicos y Constitucionales, Madrid, 2008.

DERECHOS Y LIBERTADES

ISSN: 1133-0937

Número 44, Época II, enero 2021, pp. 81-100

DOI: https://doi.org/10.20318/dyl.2021.5850 
ción como un proceso de especificación y concretización que empieza con un concepto teórico de los derechos humanos, y posteriormente se concretiza en uno de los mismos, es decir, la libertad de empresa, que toma la forma y se cristaliza en una corporación, entre otras muchas formas posibles.

La libertad de empresa tiene también sus particularidades, y una de ellas es que, a diferencia de otros derechos humanos, no hay polémica alguna sobre la consideración de que tal derecho pertenece a las corporaciones ( $u$ otras entidades $)^{13}$. En otras palabras, no hay ningún problema en compartir este derecho con los no-humanos: hay espacio para la iniciativa económica de cualquier persona o entidad que quiera ejercerlo. Por lo tanto, la libertad de empresa no solo puede constituir una base jurídica necesaria para crear una corporación, y no solo es la justificación de su existencia y su legitimización, sino que, además, cuando la corporación ya existe, este derecho se adscribe a las corporaciones de la misma manera en la que se adscribe a las personas humanas: desde esta perspectiva, o bien la libertad de empresa no es un derecho solo humano, o bien los derechos humanos pueden ser, y ya lo son, parte de la esencia de los no humanos.

En esta parte se puso en evidencia la estrecha relación que existe entre la libertad de empresa como derecho humano y la corporación que tiene el doble papel de ser ambas cosas, el objeto y el sujeto de este derecho. En la siguiente parte se pondrá en evidencia que la libertad de la empresa, que es un derecho fundamental y, además, un derecho que la corporación tiene en cuanto sujeto de Derecho, no es el único derecho que los humanos comparten con los no humanos (en este caso, corporaciones) y que la tendencia a reconocer los derechos humanos a las personas no humanas es una realidad jurídica.

\section{HUMANIZANDO CORPORACIONES: MÁS DERECHOS HUMANOS A LOS NO HUMANOS}

Hay una determinada praxis según la cual asignar personalidad jurídica a las corporaciones significa automáticamente verlas como personas humanas ${ }^{14}$. Pero la personalidad jurídica no es solo de las personas humanas. En

13 Por lo menos, ninguna polémica le consta a la autora de este trabajo, que por supuesto no quiere decir que no haya.

14 Vease, por ejemplo, J.D. CLEMENTS, Corporations Are Not People: Reclaiming Democracy from Big Money and Global Corporations, Berrett-Koehler Publishers, San Francisco, 2014. 
el Derecho -el mundo paralelo, pero estrechamente conectado con el mundo real- las personas pueden ser no solo personas humanas, sino también una miríada de otras entidades como ya se explicó brevemente con anterioridad. Es decir, la personalidad jurídica, como concepto jurídico, tiene una finalidad: la de crear personas (sujetos) que puedan ser responsables y puedan ejercer los derechos que el sistema jurídico le ha concedido. En este sentido resulta ejemplar la conclusión del juez Rozakis, que reconoció en la corporación un "organismo independiente vivo" y que cuyos derechos son protegidos por la Convención Europea de Derechos Humanos ${ }^{15}$. Claro que tal razonamiento no quiere decir que las corporaciones son personas humanas, pero sí quiere decir que, desde el punto de vista jurídico, ambos -las personas humanas y las corporaciones- son personas: muy distintas, sin duda, ya que una es natural y la otra artificial, pero, no obstante, ambas son personas y ambas tienen derechos. Una de las consecuencias de esta distinción es la responsabilidad limitada de las corporaciones y la responsabilidad ilimitada de los humanos, o la posibilidad de encarcelar a las personas humanas y no a las corporaciones. De todas formas, estas diferencias no tienen relevancia desde el punto de vista de la entidad corporativa en relación con el desempeño de su papel en la sociedad y en el Derecho ${ }^{16}$, ya que según la opinión consultiva del Tribunal Internacional de Justicia en 1949: “Los sujetos de Derecho en cualquier sistema jurídico no son necesariamente idénticos en su naturaleza o en el alcance de sus derechos, y su naturaleza depende de las necesidades de la comunidad"17. Por lo tanto, no se está apoyando la tesis de que las personas humanas y las corporaciones deban tener la misma consideración o que deban tener los mismos derechos: las diferencias son innegables $\mathrm{y}$, sin embargo, hay algunas cosas en común ${ }^{18}$, la cuales son el centro de este trabajo. Es decir, hay que buscar la respuesta a la pregunta de si puede existir algún modo para poder compartir los derechos humanos entre los humanos y no humanos sin que los mismos pierdan su valor o se vean perjudicados.

15 Vease el caso del TEDH Comingersoll SA v Portugal [GC], App no 35382/97, 2000-IV.

16 G. BARAK, Unchecked Corporate Power, Routledge, Abington, 2017, p. 32.

17 Tribunal Internacional de Justicia, "Reparation for Injuries Suffered in the Service of the United Nations: Advisory Opinion of April 11th, 1949", disponible en http://www.icj-cij.org/files/case-related/4/004-19490411-ADV-01-00-EN.pdf.

18 K. GOODPASTER, "The Concept of Corporate Responsibility", Journal of Business Ethics, núm 2, 1983, pp. 1-22. Este autor también subraya que la idea de la similitud entre las corporaciones y las personas humanas puede ser trazada a través de Platón y desde entonces ha siempre habido defensores, que han desarrollado esta idea introduciéndola en el Derecho y la teoría de las organizaciones. 
En consecuencia, ambos, los humanos y las corporaciones, pueden tener derechos similares, pero estos derechos tienen que estar "personalizados" o "moldeados" en atención a la naturaleza de cada uno.

\section{1. ¿Los derechos humanos son o no son de los humanos?}

En la hipótesis de que los individuos y las corporaciones pueden ser ambos subsumidos bajo la idea de la persona, existe una base para asignar los derechos humanos a las corporaciones. Algunas de estas asignaciones no son controvertidas, como, por ejemplo, la ya mencionada libertad de empresa, pero también el derecho a un juicio justo, el derecho a no ser condenado sin el debido procedimiento legal, o el derecho a la propiedad. Otras, al contrario, son muy controvertidas como, por ejemplo, la libertad de pensamiento y de religión ${ }^{19}$, la libertad de expresión, o la privacidad ${ }^{20}$. Las adscripciones de estos últimos derechos humanos por parte de los tribunales de EEUU, han sido interpretadas muchas veces como un proceso de humanización de la corporación, que va en paralelo con la deshumanización de los seres humanos. Por ejemplo, sobre el tema de la libertad de pensamiento y de religión en cuanto derecho reconocible a las corporaciones, el TEDH ha observado que la empresa mercantil de responsabilidad limitada, como corporación que obtiene las ganancias, no puede ni aprovecharse ni depender de los derechos del art. 9 de la Convención Europea de Derechos Humanos ${ }^{21}$.

En este choque de posiciones sobre la libertad de pensamiento, de conciencia y de religión, donde el TEDH tiene una posición diametralmente opuesta a la del Tribunal Supremo de los EEUU, para muchos autores la corporación es como "un parásito egoísta motivado solo por dinero" 22 o el

19 Se hace referencia al caso de la Corte Suprema de EEUU Burwell v Hobby Lobby, 573 US (2014).

20 Hay otros derechos que presumiblemente no pueden ser atribuidos a las corporaciones: el derecho a la vida, el derecho a formar una familia, libertad de esclavitud, ser libre de la tortura, derecho a no declarar, derecho al voto, o derecho de ser miembro de una juria popular, Véase B.L. GARRETT, "The Constitutional Standing of Corporations”, University of Pennsylvania Law Review, núm 163, 2014, pp. 95-164.

21 Art. 9 de la Convención establece la libertad del pensamiento, de conciencia y de la religión. Véase el caso de TEDH Kustannus OY Vapaa Ajattelija AB and Others v Finland App no 20471/92 del 15 de abril 1996.

22 M.K. ADDO, "Human Rights and Transnational Corporations: An Introduction”, en VV.AA., Human Rights Standards and the Responsibility of Transnational Corporations, edición de M. K. ADDO, Kluwer Law International, La Haya, 1999, p. 8. 
“obscuro señor de la globalización" 23 que demanda los derechos humanos solo para poderlos utilizar para incrementar sus ganancias, privando de tal forma a los seres humanos de su excepcionalidad jurídica ${ }^{24}$. Desde este punto de vista, el Derecho no estaría evolucionando, sino al contrario, retrocediendo, y en lugar de funcionar para el bien de los derechos humanos y, de consecuencia, de los seres humanos, funcionaría en su contra. Sin ninguna duda tales preocupaciones son fundadas y muy serias, pero la pregunta es si esa es la única interpretación posible de esos hechos y, si la respuesta a esta pregunta fuera que no, cual podría ser otra respuesta. Es decir, ¿podría el aumento de los derechos humanos reconocidos a los no humanos decir algo más que una historia de abusos contra los humanos?

No se puede poner en duda este proceso el desplazamiento de los derechos exclusivamente humanos hacia los derechos entendidos como poderes que ambos, personas humanas y no humanas (corporaciones), pueden disfrutar. Se observa que la historia de lo que las corporaciones podían hacer (y no hacer), se fue distanciando gradualmente desde los sujetos jurídicos limitados a los sujetos jurídicos emancipados ${ }^{25}, \mathrm{y}$, particularmente por lo que se refiere a TEDH, eso significa un proceso dinámico de una gradual humanización de las corporaciones ${ }^{26}$.

Aunque si el TEDH se expresó contrario a reconocer la libertad de pensamiento, de conciencia y de la religión a la corporación, dicho tribunal no ha tenido la misma opinión en relación a otros derechos humanos. Esta "humanización gradual" se observa en su jurisprudencia a través de los siguientes casos.

\subsection{Daños no pecuniarios}

El TEDH ha establecido que la corporación puede sufrir un daño no pecuniario que no sea relacionado con el sufrimiento de las personas humanas

23 J. MICKELTHWAITE y A. WOOLDGRIDGE, The Company: A Short History of a Revolutionary Idea, Phoenix, Toronto, 2005, p. 168.

24 Más sobre la excepcionalidad humana y el ser humano como sujeto y el estándar de tratamiento, véase, entre muchos, R. G. TEITEL, Humanity's Law, Oxford University Press, Oxford, 2011.

25 G. BARAK, Unchecked Corporate Power, Routledge, Abington, 2017, p. 23.

26 W. VAN DEN MUIJSENBERGH, S. REZAI, "Corporations and the European Convention on Human Rights", Pacific McGeorge Global Business \& Development Law Journal, núm 25, vol. 1, 2012, pp. 42-68. 
que trabajan en la misma, sino como una entidad que, como parte de la sociedad, tiene algunas calidades que se pueden ver afectadas, como la reputación ${ }^{27}$. Se puede ver, por lo tanto, cómo el razonamiento jurídico se desplazó de la idea de que solamente las personas humanas pueden reclamar algunos derechos. A pesar de que en el pasado los tribunales Europeos han sostenido muchas veces que los daños no pecuniarios pueden ser demandados solo por personas humanas, y, por lo tanto, las corporaciones no pueden reclamar compensaciones por pérdidas no monetarias ${ }^{28}$, la doctrina jurídica ha cambiado el rumbo y ahora tribunales tan importantes como el TEDH reconocen que las corporaciones tienen derecho a reclamarlos, siempre y cuando puedan probar que sus derechos morales que quieren proteger -derechos morales como por ejemplo el nombre, la identidad o la reputación- son compatibles con la estructura corporativa y tienen la función de conseguir salvaguardar el interés de los valores corporativos ${ }^{29}$. Por lo tanto, el hecho de que el derecho corporativo de poder reclamar este tipo de daños no depende de ningún perjuicio padecido por las personas humanas que pertenecen a la propia corporación: supone que ahora mismo este derecho está vinculado a los perjuicios que la corporación ha padecido independientemente, como entidad separada, como una persona (no obstante ser una persona no humana).

\subsection{Derecho a la privacidad}

Algo parecido pasó con el derecho a la privacidad que hasta hace poco fue considerado un derecho exclusivamente humano y por lo tanto ha sido negado a las corporaciones, sobre todo a las que reclamaron el derecho a la "privacidad personal" 30 . Pero el razonamiento ha cambiado y el TEDH ha razonado de modo distinto: si la protección de la privacidad incluye la libertad de las intrusiones en la privacidad de la propia casa, la idea de casa puede ser extendida para incluir las instalaciones comerciales ocupadas por una empresa $^{31}$. Es más, el TEDH decidió que si el derecho a la privacidad protege

27 La opinión del juez Rozakis, Tratza, Catflish y Vajić en el caso del TEDH Comingersoll SA v Portugal [GC], App no 35382/97, ECHR 2000-IV.

28 Se hace referencia a la jurisprudencia de los tribunales de Gran Bretaña, en particular al caso Firsteel Products Ltd v Anaco Ltd [1994] EWHC 80569 (QB).

${ }^{29}$ E. PALMIERINI, "I Diritti della Personalità e il Danno agli Enti Collettivi", en VV.AA. I Danni Non Patrimoniali, edición por E Navarretta, Giuffrè, Milano, 2004, p. 253.

30 Como ha establecido el Tribunal Supremo de EEUU en el caso Federal Communications Commission v ATET [2011] 562 US 397.

31 El caso de TEDH Société Colas Est and Others v France (2004) 39 EHRR 17.

ISSN: 1133-0937

DOI: https://doi.org/10.20318/dyl.2021.5850
DERECHOS Y LIBERTADES

Número 44, Época II, enero 2021, pp. 81-100 
a los individuos de la intromisión del gobierno, entonces este derecho cubre una gama entera de actividades (lícitas) en las que las personas humanas pueden estar involucradas, sin importar si estas actividades son de carácter privado o de carácter empresarial ${ }^{32}$.

La argumentación desarrollada hasta ahora está en coherencia con la idea, ya mencionada, de que las personas humanas y las corporaciones pueden compartir algunos derechos, siempre y cuando los mismos se configuren según (o a medida de) su naturaleza, sea esta naturaleza humana o corporativa. Así se puede explicar por qué, a pesar de que el TEDH reconoce el derecho a la privacidad a ambos, humanos y empresas, aún así la reconoce con mayor intensidad en el caso de personas humanas.

\subsection{Libertad de expresión}

El mismo razonamiento y enfoque metodológico se aplica en el caso de la libertad de expresión: a pesar de que esta libertad fundamental es reconocida en la Convención Europea de Derechos Humanos y otros tratados de derechos humanos internacionales, hay una distinción entre expresión comercial que se atribuye a la empresa, y expresión política de las personas humanas. En este caso dicha protección se analiza en dos niveles diferentes, la relativa a las corporaciones y la de los seres humanos, sin discriminar injustamente a ninguno de los dos ${ }^{33}$.

Es más, la jurisprudencia del TEDH explica la libertad de expresión en los casos de las corporaciones relacionando la misma con la concurrencia desleal, monopolio y violación de los derechos de los consumidores, así como con el papel de algunas corporaciones -sobre todo las relativas a los medios de la información- en contribuir y garantizar el pluralismo y proteger los valores de las sociedades democráticas ${ }^{34}$.

De esta manera no resulta contradictorio ni ontológicamente incoherente pensar que las corporaciones pueden tener derechos humanos: ahora bien,

32 A. VIŠEKRUNA, "Protection of Rights of Companies Before the European Court of Human Rights”, en VV. AA., Procedural Aspects of EU Law, edición deT. Petrašević, D. Duić, Faculty of Law Josip Juraj Strossmayer University of Osijek, Osijek, 2018, pp. 111-126.

33 Ibidem.

34 Más información sobre esos casos en CONSEJO DE EUROPA, Freedom of Expression in Europe: Case-Law Concerning Article 10 of the European Convention on Human Rights, Council of Europe Publishing, Estrasburgo, 2007.

DERECHOS Y LIBERTADES

ISSN: 1133-0937

Número 44, Época II, enero 2021, pp. 81-100

DOI: https://doi.org/10.20318/dyl.2021.5850 
éstos, si bien tienen la forma de derechos humanos, no coinciden en su contenido y peso.

Se ha visto que los derechos humanos pueden ser vistos desde la perspectiva que permite la idea de que no solo las personas humanas sino también las corporaciones pueden ser titulares de los mismos. Pero, en primer lugar, la pregunta es si es justo que las corporaciones los tengan. Más específicamente, hay que preguntarse ¿Qué ventajas supone conceder los derechos humanos a las corporaciones y cómo todo ello puede beneficiar a las personas humanas? Hasta ahora el único argumento a favor de la respuesta afirmativa a esta pregunta podría ser que garantizar la libertad de expresión a las corporaciones puede -no siempre, pero sí en algunos casos- garantizar la estabilidad de los fundamentos democráticos, pero ¿hay otros argumentos?

\section{DESAFIAR EL ESTEREOTIPO Y CAMBIAR EL PARADIGMA}

Como ya se observó al inicio de este trabajo, la idea de la corporación como explotadora de los derechos humanos no proporciona una versión completa y exhaustiva de la historia social y jurídica de la corporación. La verdad es que en la mayoría de los casos se habla de corporaciones que violan los derechos humanos, bien sea directamente, utilizando el trabajo infantil o ignorando las medidas de seguridad del lugar de trabajo por poner algunos ejemplos, o bien indirectamente, respaldando los regímenes que violan los derechos humanos como ha pasado en Nigeria, Colombia, Argentina, Birmania y muchos países más ${ }^{35}$. Teniendo estos hechos en cuenta es muy razonable pensar que es moralmente inaceptable e injusto promover y proteger los derechos humanos de las corporaciones ${ }^{36}$.

En este sentido parece que las corporaciones, no es que no puedan tener derechos humanos, sino que no se los merecen, que con los derechos humanos, las corporaciones se vuelven abusadoras de los mismos: ¿pero hay una correlación entre los derechos humanos y la buena o mala conducta? Los humanos no tienen los derechos humanos porque se comportan bien, ni

35 Más sobre esto tema, entre muchos, véase S. MICHALOWSKI (ed.), Corporate Accountability in the Context of Transitional Justice, Routledge, Abingdon, 2013.

36 Es un punto de vista muy compartido, por ejemplo, en S. TOMBS, D. WHYTE, The Corporate Criminal: Why Corporations Must Be Abolished, Routledge, Abingdon, 2015; G. BARAK, Unchecked Corporate Power, Routledge, Abington, 2017; y T. HARTMANN, Unequal Protection: The Rise of Corporate Dominance and the Theft of Human Rights, Rodale, Nueva York, 2004.

ISSN: 1133-0937

DOI: https://doi.org/10.20318/dyl.2021.5850
DERECHOS Y LIBERTADES

Número 44, Época II, enero 2021, pp. 81-100 
tampoco se les quita si se comportan mal. Como observan Muiksenbergh y Rezai, si fuera así, los derechos humanos nunca podían ser reconocidos a los criminales, a los asesinos y a los terroristas, porque son los violadores más brutales de los derechos humanos más básicos ${ }^{37}$.

Pero no es así y los derechos se reconoce a los sujetos como efecto de su existencia y su reconocimiento: el fundamento de los derechos humanos descansa en la misma consideración del ser humano, y este mismo razonamiento resulta de aplicación a los derechos humanos corporativos, como así ha afirmado el Tribunal Supremo de los EEUU, reiterando que reconocer la ficción jurídica de la corporación implica también proteger el ser humano, y reconocer derechos humanos a las corporaciones tiene también la función de proteger los mismos de las personas humanas ${ }^{38}$.

Como se puede apreciar, en este paralelismo argumental existente detrás de la humanización de la corporación como titular de derechos humanos, debe tenerse en cuenta que a la corporación no se le otorga dicho estatus por derecho propio, esto es, como una entidad que existe independientemente de los seres humanos que la componen: más bien, dicha atribución tiene lugar, por decirlo de algún modo, mediante representación, es decir, atendiendo a la forma en que la corporación afecta a la vida de dichos individuos. Por lo tanto, el sentido de este razonamiento no es que se puede "levantar el velo de la persona jurídica" (sobre la base de que los intereses protegidos no son los de la propia corporación, considerada en sí misma como entidad, sino los intereses de las personas que la constituyen en cuanto individuos): más aun, la cuestión es que, cuanto más grande sea una corporación, cuanto más lejana parezca estar de las personas o individuos que la componen o con quienes interactúa, resulta que es precisamente en atención a esas personas o a sus intereses por lo que se le atribuyen derechos.

Se puede observar la complejidad que supone el cambio de paradigma: de los derechos humanos exclusivamente para seres humanos a los derechos humanos aplicables tanto a los seres humanos como a las corporaciones. Como se habrá advertido, la humanización de las entidades corporativas se desarrolla a través de dos líneas argumentales principales: mientras que en un primer aspecto, la corporación se humaniza como una persona

37 W. VAN DEN MUIJSENBERGH, S. REZAI, "Corporations and the European Convention on Human Rights", Pacific McGeorge Global Business \& Development Law Journal, núm 25 vol. 1, 2012, pp. 42-68, p. 52.

38 El caso Burwell v Hobby Lobby, 573 US (2014) del Tribunal Supremo de EEUU.

DERECHOS Y LIBERTADES

Número 44, Época II, enero 2021, pp. 81-100
ISSN: 1133-0937

DOI: https://doi.org/10.20318/dyl.2021.5850 
considerada en sí misma, en un segundo aspecto dicha humanización tiene lugar en virtud de las personas físicas cuyos intereses son protegidos, y del mismo modo, mientras que el primer argumento o aspecto busca mostrar cómo es posible humanizar la corporación en cuanto titular de derechos, el segundo aspecto persigue explicar por qué debería ser legítimo hacerlo. La complejidad, entonces, radica estructuralmente en la múltiple naturaleza de los roles concurrentes: la corporación como tal, la corporación como entidad diseñada para proteger los intereses de sus miembros, el rol de las personas en cuanto tales y el de las personas como individuos que interactúan con la corporación en una variedad de otros roles diversos (clientes, ciudadanos, etc.).

Esto a su vez plantea la cuestión del rol de la corporación en la sociedad y explica el activismo de los derechos humanos de las personas en la sociedad. Kinley describe la relación entre las corporaciones y los activistas como una relación de desprecio, desdén mutuo, $\mathrm{y}$ "donde no era posible distinguir 'quien era el cazador y quien el cazado', porque los defensores de las corporaciones y los defensores de los derechos humanos han mostrado, por igual, instintos predatorios" 39 .

Pero, aunque el problema puede ser un conflicto de intereses, la solución puede consistir en ver que el conflicto es inherente a la multiplicidad de roles que estructuralmente interactúan en la relación, lo que puede sugerir que el potencial conflicto es, al mismo tiempo, una posibilidad para la reconciliación de los intereses involucrados.

\section{1. ¿Que pueden hacer las corporaciones por los derechos humanos?}

Hay un otro enfoque que raramente se pone de manifiesto: a lo largo de la historia las corporaciones se observa que las mismas se han convertido en "las fuerzas buenas" o de que por lo menos "han dejado de pecar tanto como antes" $^{\prime 40}$. ¿Que se quiere decir con esto?

Quizás la respuesta a esta pregunta es que las corporaciones pueden tener un impacto positivo en los derechos humanos no solamente promo-

39 D. KINLEY, "Human Rights as Legally Binding or Merely Relevant?", Commercial Law and Human Rights, edición de S. BOTTOMLEY, D. KINLEY, Ashgate, Dartmouth, 2002, pp. $25-45$, p. 25.

40 J. MICKELTHWAITE y A. WOOLDGRIDGE, The Company: A Short History of a Revolutionary Idea, Phoenix, Toronto, 2005, p. 170. 
viendo los derechos humanos directamente, sino también indirectamente, es decir, mejorando la calidad de vida, asegurando los servicios sanitarios y apoyando iniciativas e instituciones educativas, especialmente en aquellos países donde el gobierno no puede desempeñar este papel. Los ejemplos abundan ${ }^{41}$. Estas iniciativas podrían ayudar a la promoción de la situación social, económica y cultural de los derechos de los seres humanos. El impacto positivo en los derechos humanos que tienen las corporaciones en los países más pobres ha sido valorado por Meyer, quien llegó a la conclusión de que la presencia de las corporaciones en el Tercer Mundo está positivamente interrelacionada con los derechos políticos, civiles, sociales y económicos ${ }^{42}$.

Pero no hay que pensar que las corporaciones pueden ser beneficiosas solo para los derechos humanos en los países muy pobres y que no hay nada que impulsar en el panorama de los derechos humanos de las economías democráticas aparentemente fuertes. Por el contrario: también en estas últimas está teniendo lugar un cambio positivo. De hecho, Kinley argumenta que hay un cambio en la relación entre las corporaciones y los derechos humanos y que existen puntos de convergencia hacia puntos comunes ${ }^{43}$. Un ejemplo de ello es el Colectivo Emerson así como el Chan Zuckerberg Initiative ${ }^{44}$. Ambas son organizaciones filantrópicas que han elegido el tipo corporativo en lugar de constituirse como fundación porque (al menos en EEUU) ello posibilita su participación en la defensa política y poder realizar contribuciones políticas $^{45}$. En este sentido las corporaciones tienen una gran libertad de acción que les permite trabajar en beneficio de los grupos sociales vulnerables, como por ejemplo los inmigrantes, y pueden apoyar los bienes comunes trabajando para la protección del medio ambiente, la educación o un mejor acceso a los procesos democráticos. Por lo tanto, garantizar los derechos hu-

41 Uno de los numerosos ejemplos en España es el Grupo Inditex que ayuda a distintas comunidades con programas destinados a garantizar agua potable y los servicios sanitarios en Bangladesh y otros países (el programa Water.org), acceso a los servicios médicos a los trabajadores vulnerables en Marruecos (el programa Medicus Mundi) y otros. Más información

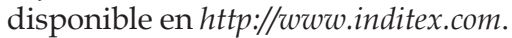

42 W. H. MEYER, "Human Rights and MNCs: Theory Versus Quantitative Analysis", Human Rights Quarterly, núm 18 vol. 2, 1996, pp. 368-397.

43 D. KINLEY, “Human Rights as Legally Binding or Merely Relevant”, cit., p. 25.

44 Disponibles respectivamente en los siguientes enlances: https://www.emersoncollective.com/, y https://chanzuckerberg.com/

${ }_{45}$ Es decir, lo que ha legitimado tan polémico caso del Tribunal Supremo de EEUU Citizens United v. Federal Election Commission, 558 U.S. 310 (2010). 
manos a las corporaciones es una forma de poderlas garantizar también a los seres humanos.

Hay otra forma en que las corporaciones contribuyen al debate global de los derechos humanos: es decir, las corporaciones pueden defender derechos humanos promoviendo los derechos humanos de los humanos frente a intromisiones o coacciones gubernamentales. Como observa Garrett, lo que enseñan las batallas judiciales de las corporaciones es que "los derechos constitucionales son mucho más fuertes cuando se deja a lado su carácter individual y se lucha por las mismas en grupo" ${ }^{46}$.

En este sentido resulta ilustrativo el asunto $\mathrm{Yukos}^{47}$. Para la corporación Yukos, el TEDH fue el lugar donde encontró una reparación contra el gobierno de la Federación Rusa ${ }^{48}$. No todos los argumentos presentados por los abogados de Yukos fueron aceptados por la TEDH, pero el asunto llamó la atención internacional por los abusos de los derechos humanos en dicho país, aportando pruebas de tales abusos. El caso Yukos también pone en evidencia que no importa el poder financiero que pueda tener una corporación, pues puede ser a su vez, igualmente, presa de abuso por parte de un gobierno. Además, la historia de Yukos enseña que los regímenes autoritativos pueden infringir no solamente los derechos humanos de los seres humanos, sino también los derechos humanos de las corporaciones, debiendo señalar que, si una poderosa corporación fue fácil y rápidamente desmantelada, hay que preguntarse qué les ocurre a los disidentes, a los oponentes políticos y a otras personas o colectivos que alzan sus voces contra las instituciones políticas y cuestionan sus actuaciones.

Un razonamiento similar se puede aplicar a aquellos supuestos donde las corporaciones han formulado reclamaciones en materia de protección de la privacidad: el objetivo general de la protección de la privacidad en la sede del TEDH es la protección de las personas y de las corporaciones frente a las injerencias indebidas. No obstante, las actividades personales y de negocio suelen estar entrelazadas, lo que dificulta poder trazar una línea de separación entre ellas. Por lo tanto el derecho a la privacidad de las corporaciones

46 B. L. GARRETT, "The Constitutional Standing of Corporations", University of Pennsylvania Law Review, núm 63, 2014, pp. 95-164, p. 164.

47 El caso de TEDH OAO Neftyanaya Kompaniya Yukos v Russia App no 14902/04, del 31 julio 2014.

48 Para más detalles sobre este caso, véase W. VAN DEN MUIJSENBERGH, S. REZAI, "Corporations and the European Convention on Human Rights", cit. 
puede servir como un escudo para preservar un derecho individual a la privacidad, y en particular el de sus empleados.

Es por este motivo que se ha defendido la posición de que "si las demandas corporativas pueden garantizar el uso efectivo de los derechos y los principios de la Convención Europea de los Derechos Humanos por parte de la sociedad, el sistema de valores de la Convención aceptará dichas demandas" ${ }^{49}$. En este sentido, las reclamaciones corporativas de los derechos humanos se contemplan como instrumentos de promoción de los derechos humanos de los individuos. Más aun, como las entidades tienen derechos humanos, las corporaciones están incrementando considerablemente la función, alcance y significado de estos derechos: ser titular de derechos humanos no es solamente exigir a los demás su respeto, sino también respetar recíprocamente los derechos de los demás. Por lo tanto, si las corporaciones y los individuos están obligados a respetar y proteger los derechos humanos, entonces también se reconoce dicho respeto y protección no solo a los seres humanos sino también a las corporaciones. Esto se manifiesta en el llamado principio de proyección moral de Goodpaster, en cuya virtud hay que exigir y pretender de las corporaciones lo mismo que se exige y se pide de los seres humanos ${ }^{50}$ : según el razonamiento expuesto, el principio debería funcionar en el sentido inverso, es decir, pedir a los individuos (legisladores, políticos, ejecutivos) el mismo esfuerzo que se pretende a las corporaciones en la protección de los derechos humanos.

Cambiar el paradigma que supone pasar de la consideración de las corporaciones en cuanto infractoras de los derechos humanos, a pasar a considerarlas también como potenciales víctimas de dichas infracciones es una invitación a contemplar a la corporación como un "organismo vivo independiente" 51 , de forma que sea considerada como "parte integrante de la sociedad" y "como una persona social y política a través de la cual puedan ser mejoradas sus contribuciones a la sociedad" 52 . Algunos autores han sugerido que esto podría conducirnos a una iniciativa conjunta de derechos humanos para Europa, destacando las especificidades propias de la persona

\footnotetext{
$49 \quad$ Ibidem, p. 57.

50 GOODPASTER, "The Concept of Corporate Responsibility”, cit.

51 La opinión de juez Rozakis, Tratza, Catflish y Vajić en el caso del TEDH Comingersoll SA v Portugal [GC], App no 35382/97, ECHR 2000-IV.

52 ADDO, "Human Rights and Transnational Corporations: An Introduction", cit., p. 8.
}

DERECHOS Y LIBERTADES

Número 44, Época II, enero 2021, pp. 81-100
ISSN: 1133-0937

DOI: https://doi.org/10.20318/dyl.2021.5850 
corporativa $^{53}$. Dicha iniciativa podría ampliar y reinterpretar los derechos humanos existentes adaptándolos a la naturaleza legal de las personas, contribuyendo también a la teoría jurídica de la personalidad de la corporación. Obviamente, no se necesita un tratado separado, pero un debate al respecto resultaría beneficioso ya que permitiría contemplar los derechos humanos desde una perspectiva que podría revelar la historia completa de las conexiones entre los individuos y las corporaciones.

\section{CONCLUSIONES}

Hace algún tiempo se está debatiendo la cuestión de nuevos derechos que se debería reconocer a los animales, y actualmente la Naturaleza, en sus diversas manifestaciones como son los ríos y otras formaciones naturales, así como los pasajes naturales, está siendo reconocida como titular de una personificación legal ${ }^{54}$. Lo que hace posible conceder derechos humanos a los no humanos, según Garrett, es que muchos de estos derechos "no son estrictamente personales y se les puede aplicar en general, sea son concebidos como los deberes del Estado o los privilegios contra el mismo" ${ }^{55}$. Por eso mismo no debe sorprender que los derechos humanos no sean concedidos solamente para los humanos, sino que también pueden ser otorgados a los no humanos, es decir, a las corporaciones.

Hay dos historias sobre la relación entre los derechos humanos y a las corporaciones: una de ellas destaca la trayectoria histórica de las corporaciones como infractoras de derechos humanos. La otra, por contra, contempla a las corporaciones como una fuerza potencial para el bien de los derechos humanos. Observar esta historia en su integridad es lo que ayuda entender su complejidad.

Desde luego la cuestión es mucho mas difícil puesto que, como ya se señaló, las corporaciones operan en la sociedad: sus acciones, por tanto, tienen consecuencias sociales, y los intereses en juego plantean determinadas cues-

53 VIŠEKRUNA, "Protection of Rights of Companies Before the European Court of Human Rights", cit., p. 124.

54 La personalidad jurídica ha sido reconocida al área Te Urewera en Nueva Zelanda, y a algunos ríos: el río Whanganui (Nueva Zelanda), los ríos Ganges and Yamuna (Índia), y el rio Atrato (en Colombia). Más sobre esto en L. CANO PECHARROMAN, "Rights of Nature: Rivers That Can Stand in Court", Resources, núm 7 vol. 13, 2018, pp. 1-14.

55 GARRETT, “The Constitutional Standing of Corporations”, cit., p. 161.

ISSN: 1133-0937

DOI: https://doi.org/10.20318/dyl.2021.5850
DERECHOS Y LIBERTADES

Número 44, Época II, enero 2021, pp. 81-100 
tiones de índole práctica y moral cuya solución no es siempre tan evidente. Por lo tanto, hay que encontrar la manera de conceder derechos humanos a las corporaciones de modo tal que los beneficios lleguen a los empleados, sin menoscabar los intereses de la sociedad.

\author{
Migle Laukyte \\ Facultad de Derecho \\ Universitat Pompeu Fabra \\ c/ Ramon Trias Fargas, 25-27 \\ 08005 Barcelona \\ e-mail: migle.laukyte@upf.edu
}

